\title{
Etiology of Epiphora
}

\author{
Jeong Min Lee, Ji Sun Baek \\ Department of Ophthalmology, Kim's Eye Hospital, Konyang University College of Medicine, Seoul, Korea
}

\begin{abstract}
Purpose: This study aimed to analyze the common causes of epiphora in Korean patients and their response to subsequent management.

Methods: We retrospectively analyzed the medical records of 180 patients who visited Kim's Eye Hospital for epiphora between December 2017 and January 2019. This study included 320 eyes of 180 patients.

Results: In the 320 eyes of 180 patients, the most common etiology of epiphora was reflex tearing due to dry eye syndrome, which occurred in 167 eyes (52.19\%). The other etiologies of epiphora included anatomical abnormality (68 eyes, 21.25\%), multifactorial (60 eyes, 18.75\%), functional epiphora (14 eyes, 4.38\%), ocular surface disease (seven eyes, $2.19 \%$ ), and eyelid abnormality (four eyes, $1.25 \%$ ).

Conclusions: The most common etiology of epiphora in Korean patients was reflex tearing due to dry eye syndrome, followed by lacrimal passage abnormality, multifactorial, functional epiphora, anterior segment disease, and eyelid malposition. Most patients with reflex tearing reported improvement in their symptoms after lubrication.
\end{abstract}

Key Words: Dry eye syndromes, Lacrimal apparatus diseases, Lacrimal drainage system abnormality, Tearing

Epiphora or tearing is defined as excessive watering of the eye. It is a common complaint among referrals to oculoplastic clinics. The process of tearing includes several steps such as formation in the lacrimal gland, spreading through eye blinking, vaporization from the ocular surface, and draining through the nasolacrimal duct. Abnormalities in any of these steps can cause epiphora [1]. Numerous etiologies can lead to an imbalance between tear formation and tear loss, leading to epiphora. Excess tear production directly leads to tearing, whereas tear loss stimulates re-

Received: May 3, 2021 Final revision: June 6, 2021

Accepted: July 4, 2021

Corresponding Author: Ji Sun Baek, MD. Department of Ophthalmology, Kim's Eye Hospital, Konyang University College of Medicine, 136 Yeongsin-ro, Yeongdeungpo-gu, Seoul 07301, Korea. Tel: 82-2-2639-7518, Fax: 82-2-2671-6359, E-mail: yangchi100@kimeye.com flective and spontaneous tear production, which may also result in epiphora. Most imbalances in tear quantity are caused due to nasolacrimal duct stenosis and obstruction (at a different level of lacrimal outflow system), eyelid malposition, lacrimal pump failure, and dry eye with reflex tearing $[2,3]$. However, a combination of these causes has been identified in some cases. Thus, a multipronged treatment approach is necessary to resolve the symptoms, and assessment of the response to treatment may be needed to confirm the diagnosis. Our understanding of epiphora is evolving over time; nevertheless, the exact pathophysiological mechanisms underlying the development of epiphora are unknown.

Previous studies have described the causes and outcomes of epiphora management, and concrete data on the etiology of epiphora has also been reported. In a study by Mainville and Jordan [4], 48.7\% patients with tearing had a lacrimal 
system block and $40 \%$ patients had dry eye syndrome. In a study by Sibley et al. [5], 31.8\% subjects had nasolacrimal duct obstruction, which was followed by the development of dry eye syndrome with secondary reflex tearing in 29.2\% patients. However, there are few articles describing the causes of epiphora in Korean patients.

Therefore, the present study aimed to analyze the common causes of epiphora in Korean patients and patient response to subsequent management. The study outcomes could serve as a benchmark for evaluating the effectiveness of epiphora management in Korean patients. In addition, most recent studies analyzing the etiology of epiphora have mainly been conducted at tertiary hospitals managing patients with high disease severity. However, in this study, we analyzed the etiology of epiphora in a secondary hospital, which is relatively easy to visit than a tertiary hospital. Through this study, we aimed to investigate the more fundamental and widespread causes of epiphora.

\section{Materials and Methods}

The study was approved by the institutional review board of Kim's Eye Hospital, Seoul, Korea (2020-04-009). Considering the retrospective nature of the study and the use of deidentified patient data, the written informed consent was waived. All procedures were performed in accordance with the Declaration of Helsinki guidelines.

We retrospectively analyzed and collected data from the medical records of 180 patients who visited the Oculoplastic Clinic at Kim's Eye Hospital for epiphora between December 2017 and January 2019. This study included 320 eyes of 180 patients. The patients with epiphora underwent detailed interviews about their symptoms, including questions regarding the duration of epiphora, accompanying symptoms, and aggravating and relieving factors. Further, the patients were asked detailed questions regarding the symptoms associated with dry eye syndrome (including burning, grittiness, and itching sensation) and lacrimal passage abnormalities (including questions regarding tearing all the times, regardless of the time and place). All patients who visited the Oculoplastic Clinic underwent ocular surface examination using a slit lamp. A single ophthalmologist (JSB) verified the eyelid and punctal position, tear meniscus height, tear film break-up time, and fluorescein dye retention test results. If a lacrimal passage ab- normality was suspected on slit-lamp examination, dacryocystography (DCG) was performed after the examination using a syringe and probe to diagnose lacrimal pathway obstruction. We reviewed the patient charts and organized the syringing, probing, and DCG findings systematically.

We subclassified the etiologies of epiphora according to the primary pathophysiological cause, including the following: (1) dry eye syndrome with reflex tearing, (2) anterior segment disease (such as trichiasis, pterygium, pingueculae, and conjunctivochalasis), (3) eyelid malposition (such as horizontal lower eyelid laxity and ectropion), (4) lacrimal passage abnormality (such as punctal or canalicular obstruction, nasolacrimal duct stenosis and obstruction, (5) functional epiphora, and (6) multifactorial epiphora (such as simultaneous nasolacrimal duct obstruction and dry eye syndrome with or without lower eyelid laxity).

A diagnosis of reflex tearing associated with dry eye syndrome was made if a combination of the following were present: symptoms associated with dry eye syndrome, reduced tear meniscus height and tear film breakup time ( $<10$ seconds), signs of meibomian gland dysfunction, and signs of ocular surface dryness (such as positive corneal fluorescein staining). Although the ocular surface is not dry, reflex tearing may occur when persistent irritation is affecting the anterior segment of the eye, such as trichiasis, pterygium, pingueculae, and conjunctivochalasis. In addition, eyelid malposition can cause epiphora. All patients underwent lacrimal syringing and probing. A diagnosis of canalicular obstruction was made based on the occurrence of a soft stop on probing, and nasolacrimal duct obstruction was diagnosed when there was a lack of recovery of irrigation fluid in the nose. The results were confirmed by DCG. Functional epiphora was diagnosed when a patient had symptoms of tearing and a corresponding high tear meniscus height. However, all other causes of epiphora were excluded, including cases with no signs of dry eye syndrome, no pathological findings in the anterior segment and on eyelid position, no abnormalities detected during syringing and probing, and no abnormal DCG results. We included cases with no organic problems with currently existing techniques, possibly due to issues related to functional epiphora. We guess that a defective lacrimal pump mechanisms might be responsible for functional epiphora. If more than one component was identified as an etiological factor, the case was classified as multifactorial. 
After diagnosis, appropriate treatment was administered, the patients were followed up, and all the results were recorded.

\section{Results}

Among the 180 patients, 53 were males and 127 were females. Their mean age was $60.46 \pm 12.32$ years (range, 17 84 years). The mean duration of symptoms was $36.10 \pm$ 62.49 months (range, $0.3-480$ months). Forty patients complained of monocular symptoms and 140 patients complained of symptoms in both eyes. The patient demographic information is summarized in Table 1.

In the 320 eyes of 180 patients, the most common etiology of epiphora was dry eye syndrome with reflex tearing, which was observed in 167 eyes (52.19\%). The other etiologies of epiphora were lacrimal passage abnormality (68 eyes, $21.25 \%$ ), multifactorial epiphora (60 eyes, 18.75\%), functional epiphora (14 eyes, 4.38\%), anterior segment disease ( 7 eyes, $2.19 \%$ ), and eyelid malposition ( 4 eyes, $1.25 \%$ ), as summarized in Table 2.

All patients with dry eye syndrome with reflex tearing (167 eyes of 89 patients) were instructed to use artificial tears several times a day. Patients were then scheduled for a follow-up visit 4 weeks later. Among the 89 patients, 27 returned to the clinic on their scheduled date. Among the 27 patients (51 eyes), 25 patients (47 eyes, 92.16\%) reported improvement in epiphora, while two patients (4 eyes, (7.84\%) reported no improvement with treatment.

We further analyzed epiphora in eyes with lacrimal passage abnormalities. It was confirmed that 68 eyes had lacrimal passage stenosis or obstruction, resulting in epiphora. Nasolacrimal duct obstruction (28 eyes, $41.18 \%$ ) was the most common cause, followed by nasolacrimal duct stenosis (27 eyes, $39.71 \%$ ), common canalicular obstruction (nine eyes, 13.24\%), and punctal obstruction (four eyes, 5.88\%) (Table 3).

The 60 eyes that were diagnosed with multifactorial epiphora were treated based on their most dominant cause. The most common cause of multifactorial epiphora was a combination of dry eye syndrome with reflex tearing and lacrimal passage abnormality, which was observed in 29 eyes (48.33\%). Fourteen eyes (23.33\%) had dry eye syndrome and 15 eyes $(25.0 \%)$ had predominantly lacrimal passages abnormality. In 12 patients $(20 \%)$, the three prob-
Table 1. Demographic information of patients with epiphora

\begin{tabular}{lc}
\hline Demographic factor & Value \\
\hline Age (yr) & $60.46 \pm 12.32$ \\
Sex (male : female) & $53(29.4): 127(70.6)$ \\
Duration of symptom (mon) & $36.10 \pm 62.49$ \\
Side of tearing eye (monocular : binocular) & $40(22.2): 140(77.8)$ \\
\hline
\end{tabular}

Values are presented as mean \pm standard deviation of number (\%).

Table 2. Etiology of epiphora

\begin{tabular}{lc}
\hline Etiology & Value \\
\hline Dry eye syndrome with reflex tearing & $167(52.19)$ \\
Lacrimal passage abnormality & $68(21.25)$ \\
Multifactorial epiphora & $60(18.75)$ \\
Functional epiphora & $14(4.38)$ \\
Anterior segment disease & $7(2.19)$ \\
Eyelid malposition & $4(1.25)$ \\
\hline
\end{tabular}

Values are presented as number of eyes (\%).

Table 3. Eyes with epiphora and lacrimal passage abnormality

\begin{tabular}{lc}
\hline Lacrimal passage abnormality & Value \\
\hline Nasolacrimal duct obstruction & $28(41.18)$ \\
Nasolacrimal duct stenosis & $27(39.71)$ \\
Common canalicular obstruction & $9(13.24)$ \\
Punctal obstruction & $4(5.88)$ \\
\hline
\end{tabular}

Values are presented as number of eyes (\%).

lems were concurrently observed. Twenty-eight eyes were multifactorial epiphora with dry eye syndrome with reflex tearing as the most problematic factor; hence, these patients were treated for dry eye syndrome, including instillation of artificial tears. Eight of 28 eyes were lost to follow-up, and the remaining 20 eyes showed improvement in tearing symptoms after treatment. The causes of multifactorial epiphora are shown in Table 4.

\section{Discussion}

Epiphora is one of the most common ocular problems encountered in oculoplastic clinics. Nevertheless, few studies have described the etiologies of epiphora in Korean patients. Therefore, we aimed to investigate the common causes of epiphora in Koreans as well as their response to subsequent management. We found that the most common 
Table 4. Causes of multifactorial epiphora

\begin{tabular}{lc}
\hline Causes of multifactorial epiphora & Value \\
\hline Lacrimal passage abnormality $>$ dry eye syndrome with reflex tearing & $15(25.0)$ \\
Lacrimal passage abnormality $>$ eyelid malposition & $6(10.0)$ \\
Lacrimal passage abnormality $>$ anterior segment disease & $1(1.67)$ \\
Dry eye syndrome with reflex tearing $>$ lacrimal passage abnormality & $14(23.33)$ \\
Dry eye syndrome with reflex tearing $>$ anterior segment disease & $6(10.0)$ \\
Dry eye syndrome with reflex tearing $>$ eyelid malposition & $2(3.33)$ \\
Eyelid malposition $>$ dry eye syndrome with reflex tearing & $2(3.33)$ \\
Anterior segment disease $>$ dry eye syndrome with reflex tearing & $2(3.33)$ \\
Dry eye syndrome with reflex tearing $>$ lacrimal passage abnormality > anterior segment disease & $4(6.67)$ \\
Lacrimal passage abnormality $>$ dry eye syndrome with reflex tearing > eyelid malposition & $2(3.33)$ \\
Lacrimal passage abnormality $>$ anterior segment disease > dry eye syndrome with reflex tearing & $2(3.33)$ \\
Lacrimal passage abnormality $>$ eyelid malposition $>$ dry eye syndrome with reflex tearing & $2(3.33)$ \\
Dry eye syndrome with reflex tearing $>$ anterior segment disease $>$ lacrimal passage abnormality & $2(3.33)$
\end{tabular}

Values are presented as number of eyes (\%).

etiology was reflex tearing due to dry eye syndrome, followed by lacrimal passage abnormality, multifactorial disease, functional disease, anterior segment disease, and eyelid malposition.

In general, when consulting patients with epiphora, the first thing that clinicians should consider is the possibility of anatomical abnormalities in the lacrimal passage. Tears start from the eyelid puncta and flow to the inferior nasal meatus along the lacrimal passage. Therefore, stenosis or obstruction can be observed at different levels of this lacrimal passage. Although nasolacrimal duct obstruction may be congenital, it can also develop in the second half of the patient's life. Most acquired cases of obstructed lacrimal passage occur due to chronic inflammation of the lacrimal sac [6]. In previous reports, cases of nasolacrimal duct obstruction could be congenital and acquired, and their frequencies were $10.1 \%$ to $33.3 \%$ [7-9].

Anatomical abnormalities the of lacrimal passage have been reported as the most common cause of epiphora in previous studies $[4,5]$. One of the first studies to report this was conducted by Mainville and Jordan [4], who found that lacrimal passage abnormality was associated with epiphora in $48.7 \%$ cases, followed by dry eye-associated reflex tear secretion in $40 \%$ cases. Sibley et al. [5] reported that partial or complete lacrimal obstruction is the most common cause of epiphora (31.8\%), followed by dry eye syndrome with secondary reflex tearing $(29.2 \%)$. Williams et al. [9] reported nasolacrimal duct obstruction (obstruc- tion at any spot within the nasolacrimal passage) was the most common cause of epiphora (33.3\%). However, anatomical abnormalities, including lacrimal passage obstruction, were the second most common etiology of epiphora in our study, accounting for $21.25 \%$ of cases of epiphora. Furthermore, reflex tearing due to dry eye syndrome was the most common cause of epiphora, with a rate of $52.19 \%$. The reason for the higher proportion of reflex tearing due to dry eye syndrome in this study than that in other studies is that Kim's Eye Hospital, where this study was conducted, is a secondary hospital; hence, patients with relatively low disease severity could visit easily. In addition, at Kim's Eye Hospital, if the patients visit with a complaint of epiphora, the reception desk usually lets the patients consult the oculoplastic practice doctor. This can lead to a higher proportion of patients with dry eye syndrome and reflex tearing. The percentage of multifactorial epiphora in this study was also higher than that reported in other studies. This may be because, in this study, multifactorial epiphora included anatomical abnormalities such as lacrimal passage obstruction or stenosis.

Dry eye syndrome and its related reflex tearing are common ophthalmic conditions [10-13]. Although our understanding of dry eye syndrome and reflex tearing is limited, a recent study has provided significant insights into the mechanisms underlying the development of epiphora. Conventionally, the causes of dry eye syndrome are classified as aqueous tear deficiency or loss of evaporation. A recent 
study has suggested that early detection of dry eye syndrome includes tear hyperosmolarity and tear film instability [11]. The instability of the tear film results in loss of evaporation and increased tear osmolality. Chronically elevated tear osmolarity increases the expression of several cytokines, resulting in inflammation of the ocular surface and reduced function of goblet cells, which may induce epithelial injury. This makes the tear film more unstable, and the cycle is continuous. During the early stages of dry eye syndrome, neurosensory stimuli from the ocular surface may lead to increased lacrimal gland tear secretion to lower tear film osmolarity, which results in reflex tearing. These reactions have also been observed in meibomian gland dysfunction [14].

Patients diagnosed with dry eye syndrome were prescribed artificial tears and anti-inflammatory ophthalmic solutions for blepharitis. Of the 167 eyes with dry eye syndrome, 116 eyes were lost to follow-up and 47 eyes showed significant improvement or resolution of their symptoms. No improvement was found in four eyes. Improvement after lubrication and use of anti-inflammatory solutions was observed in most eyes that were followed up (92.16\%). Mainville and Jordan [4] reported a significant improvement in reflex tearing with artificial tears in $67.0 \%$ patients with dry eye syndrome, no improvement in $6.7 \%$ patients, and no follow-up in $26.7 \%$ patients. Excluding patients lost to follow-up, their study results are similar to our study results. The reason why many patients were lost to follow-up in our study is likely due to the fact that Kim's Eye Hospital is a secondary hospital; hence, it is easy for patients to visit the hospital without making an appointment in advance. Patients tend to not visit hospitals regularly once their symptoms start to improve.

When investigating the association between sex and epiphora, we found that females are more vulnerable to epiphora than men. Similar to our findings, several recent studies have shown that epiphora is more common in females than in males and is often bilateral [8]. Acquired nasolacrimal duct obstruction is the most common cause of epiphora in middle-aged or older females [15]. In a study by Woog [16], 73\% patients with nasolacrimal duct obstruction were females. It is possible that the narrowness of the nasolacrimal canal and the sharp angle between the bony canal and the base of the nasal floor predispose females to chronic inflammation of the nasolacrimal drainage system [17].
Epiphora can become chronic if left untreated. In one study, the average duration of symptoms was 41.1 months. When the etiologies were examined individually, it was observed that the longest symptom duration (61.9 months) was due to eyelid malposition and the shortest symptom duration (28.5 months) was due to dry eye [5]. In our study, the average duration of the symptoms was 36.1 months. Two cases had the longest symptom duration of 480 months, both of which were related to nasolacrimal duct obstruction. However, cases with the shortest symptom duration ( 0.3 months) were due to reflex tearing due to dry eye syndrome.

This study has several limitations. The most significant limitation is that it was a single-institution retrospective study with a relatively small sample size including Asians. Therefore, future large-scale, multicenter, prospective studies are necessary to validate the study findings. In addition, since this study was conducted at a secondary hospital, the study results may be different from those reported by studies conducted at tertiary hospitals, which have a higher proportion of severe cases.

In conclusion, the most common cause of epiphora in Korean patients was dry eye syndrome with reflex tearing, followed by lacrimal passage abnormality, multifactorial etiology, functional epiphora, anterior segment disease, and eyelid malposition. Fortunately, most patients with reflex tearing due to dry eye syndrome reported the recovery of symptoms after lubrication. Thus, a comprehensive evaluation is necessary to find the cause of epiphora and introduce proper management.

\section{Conflict of Interest}

No potential conflict of interest relevant to this article was reported.

\section{Acknowledgements}

This study was supported by the Kim's Eye Hospital Research Center. 


\section{References}

1. Tsubota K. Tear dynamics and dry eye. Prog Retin Eye Res 1998;17:565-96.

2. Vick VL, Holds JB, Hartstein ME, Massry GG. Tarsal strip procedure for the correction of tearing. Ophthalmic Plast Reconstr Surg 2004;20:37-9.

3. Liarakos VS, Boboridis KG, Mavrikakis E, Mavrikakis I. Management of canalicular obstructions. Curr Opin Ophthalmol 2009;20:395-400.

4. Mainville N, Jordan DR. Etiology of tearing: a retrospective analysis of referrals to a tertiary care oculoplastics practice. Ophthalmic Plast Reconstr Surg 2011;27:155-7.

5. Sibley D, Norris JH, Malhotra R. Management and outcomes of patients with epiphora referred to a specialist ophthalmic plastic unit. Clin Exp Ophthalmol 2013;41:2318 .

6. A VP, Patil SS, Koti SV, et al. Clinico-bacteriological study of chronic dacryocystitis cases in northern Karnataka, India. J Clin Diagn Res 2013;7:2502-4.

7. Bukhari A. Etiology of tearing in patients seen in an oculoplastic clinic in Saudi Arabia. Middle East Afr J Ophthalmol 2013;20:198-200.

8. Nemet AY. The etiology of epiphora: a multifactorial issue. Semin Ophthalmol 2016;31:275-9.

9. Williams B, Johnson D, Hurst J, Kratky V. Patterns and causes of epiphora referrals to a tertiary oculoplastic practice. Can J Ophthalmol 2014;49:180-2.
10. The definition and classification of dry eye disease: report of the Definition and Classification Subcommittee of the International Dry Eye WorkShop (2007). Ocul Surf 2007;5:75-92.

11. Fox RI, Howell FV, Bone RC, Michelson P. Primary Sjogren syndrome: clinical and immunopathologic features. Semin Arthritis Rheum 1984;14:77-105.

12. Lin PY, Tsai SY, Cheng CY, et al. Prevalence of dry eye among an elderly Chinese population in Taiwan: the Shihpai Eye Study. Ophthalmology 2003;110:1096-101.

13. Schaumberg DA, Sullivan DA, Buring JE, Dana MR. Prevalence of dry eye syndrome among US women. Am J Ophthalmol 2003;136:318-26.

14. Shimazaki J, Sakata M, Tsubota K. Ocular surface changes and discomfort in patients with meibomian gland dysfunction. Arch Ophthalmol 1995;113:1266-70.

15. McNab AA. Lacrimal canalicular obstruction associated with topical ocular medication. Aust $N Z$ J Ophthalmol 1998;26:219-23.

16. Woog JJ. The incidence of symptomatic acquired lacrimal outflow obstruction among residents of Olmsted County, Minnesota, 1976-2000 (an American Ophthalmological Society thesis). Trans Am Ophthalmol Soc 2007;105:64966.

17. Shigeta K, Takegoshi H, Kikuchi S. Sex and age differences in the bony nasolacrimal canal: an anatomical study. Arch Ophthalmol 2007;125:1677-81. 